\title{
Molecular evidence that aphid-transmitted Alpinia mosaic virus is a tentative member of the genus Macluravirus
}

\author{
Brief Report \\ R. F. Liou, H. Z. Yan, and J. L. Hong \\ Department of Plant Pathology, National Taiwan University, Taipei, Taiwan \\ Received October 17, 2002; accepted January 17, 2003 \\ Published online March 21, 2003 (C) Springer-Verlag 2003
}

Summary. Alpinia mosaic virus (AlpMV), once assigned to the genus Potyvirus, infects primarily plants of the ginger family. To seek molecular evidence for correct classification of this virus, a cDNA clone corresponding to the $3^{\prime}$ portion of the AlpMV genome was obtained by reverse transcriptase-PCR and TA cloning. The authenticity of the cDNA clone was confirmed by expression of the coat protein (CP) in E. coli followed by immunoblot analysis. Sequence analysis indicated that, in contrast to its low identity with all the other genera of the family Potyviridae, the deduced amino acid sequence of AlpMV CP was $42.9 \sim 61.9 \%$ identical to members of the genus Macluravirus. Phylogenetic analysis also demonstrated that the AlpMV CP clustered with those of Cardamom mosaic virus and Chinese yam necrotic mosaic virus. These results indicate that AlpMV should be classified as a tentative species within the genus Macluravirus rather than Potyvirus as proposed previously.

Plants of the ginger family, Zingiberaceae, have long been used as condiments, dyes, perfumes, spices, and vegetables. Moreover, owing to their beautiful foliage and flowers, some species of the ginger family, especially Curcuma alismatifolia, have become floricultural crops of considerable economical importance in some countries in the past few years. Viruses that have been reported to infect plants of the ginger family include Cardamom mosaic virus (CdMV) [11], Cardamom chirke virus, Cardamom foorkey virus [4], Cucumber mosaic virus (CMV) [18], and Ginger chlorotic fleck virus (GCFV) [19]. Recently, a new virus known to infect primarily plants of the ginger family was described by Chen and Hong [5] in Taiwan and designated as Alpinia mosaic virus (AlpMV). It has flexuous filamentous particles with a modal length of $700 \sim 750 \mathrm{~nm}$ and is transmissible only 
by the banana aphid, Pentalonia nigronervosa, in a non-persistent manner. Plants which are known to be host of AlpMV include ginger (Zingiber officinale), Alpinia formosana, Alpinia purpurata, Curcuma alismalifolia, Hedychium hybridum, and Phaeomeria magnifica. After infection, numerous light green stripes appear on the leaves of infected plants, and pinwheel-shaped and laminated inclusion bodies can be detected in them. Accordingly, AlpMV has been classified under the family Potyviridae and the genus Potyvirus [5, 9]. Recently, the genus Macluravirus was established for species which, although aphid transmissible, contain coat protein genes significantly different from those of the genus Potyvirus. Macluravirus has two definitive members: Maclura mosaic virus (MacMV) and Narcissus latent virus (NLV) [3]. In addition, two viruses were recently proposed to be tentative members of Macluravirus, namely Cardamom mosaic virus (CdMV) [12] and Chinese yam necrotic mosaic virus (ChYNMV) [14], based essentially on the sequence data. To determine the classification of AlpMV, the nucleotide sequence of the 3 '-terminal region of this virus was cloned and analyzed. The results obtained indicate that AlpMV is a tentative new member of the genus Macluravirus and not Potyvirus as proposed previously.

Virus was purified directly from diseased Alpinia formosana showing the characteristic mosaic pattern according to Gonsalves et al. [11] and Chen and Hong [5]. Viral RNA was isolated from purified virus particles by proteinase $\mathrm{K}$ digestion, phenol chloroform extraction and ethanol precipitation [7]. Following reverse transcription primed with oligo(dT), viral cDNA was amplified by PCR using oligo(dT) and a potyvirus universal primer, which was provided by Dr. Y. C. Chang (Department of Plant Pathology, National Taiwan University), as the downstream and upstream primers, respectively. Each reaction $(20 \mu \mathrm{l})$ consisted of $10 \mathrm{ng}$ of viral cDNA, $1.25 \mu \mathrm{M}$ of oligonucleotide primers, $0.2 \mathrm{mM}$ dNTP, $1 \times$ PCR buffer, and $1 \mathrm{U}$ of DyNazyme ${ }^{\mathrm{TM}}$ II DNA polymerase (Finnzymes, Finland). PCR was performed by denaturation at $94^{\circ} \mathrm{C}$ for $5 \mathrm{~min}$, followed by 25 cycles of $95^{\circ} \mathrm{C} / 45 \mathrm{sec}, 55^{\circ} \mathrm{C} / 1 \mathrm{~min}, 72^{\circ} \mathrm{C} / 2 \mathrm{~min}$, and a final 10 -min extension at $72^{\circ} \mathrm{C}$ in a thermocycler (GeneAmp PCR System 2400, Perkin elmer). After separation of the amplified product on a $1 \%$ agarose gel, DNA bands of expected size were collected from the gel using the Geneclean III kit (Bio101) and cloned into pGem T-easy (Promega). The nucleotide sequence was determined on both strands of DNA using the BigDye terminator cycle sequencing ready reaction kit and an autosequencer (Applied Biosystems, model 310). Sequence was analyzed using programs in the GCG software package (Genetics Computer Group, Wisconsin Package Version 10.0).

The coat protein $(\mathrm{CP})$ of AlpMV was expressed using the pQE31 bacterial expression system (Qiagen). A DNA fragment containing the entire putative CP was amplified from the cDNA clone (alpcp-1) using the T7 promoter primer (5'-TAA TAC GAC TCA CTA TAG GG-3') and alpexp1 (5'-CCA AGC TTG GTT AAT GTA GCG TTG CAC GCG-3'), which comprised the complement of the last 20 nucleotides of the open reading frame (ORF), including the termination codon and, immediately downstream, a HindIII site for the purpose of cloning. The amplified DNA fragment was gel purified, digested with BamHI and HindIII, and cloned into 
the expression vector $\mathrm{pQE} 31$ to generate the recombinant plasmid clone pExcp12. The BamHI site was located at position 531 to 536 of the AlpMV cDNA and used to facilitate in frame ligation of the CP ORF with the translation initiation codon ATG in pQE31. For expression of the CP, pExcp12 was transformed into E. coli strain M15[pREP4]. Expression of the recombinant protein by E. coli was induced by addition of $1 \mathrm{mM}$ IPTG (isopropyl-thio- $\beta$-D-galactopyranoside).

After IPTG induction, E. coli cells were harvested by centrifugation, and the pellet was mixed with a sample buffer [final concentration: $0.06 \mathrm{M}$ Tris- $\mathrm{HCl}$, $\mathrm{pH} 6.8,2 \%$ SDS (w/v), $10 \%$ glycerol, $5 \% \beta$-mercaptoethanol (v/v), and $0.1 \%$ bromophenol blue], and the proteins were analyzed by $12 \%$ SDS polyacrylamide gel electrophoresis (PAGE). The separated proteins were blotted onto PVDF membrane (Osmonics) using a Multiphor II Electrophoresis Unit (Amersham Pharmacia) as the transfer apparatus. The immunoblot was blocked for $1 \mathrm{~h}$ in a blocking solution (Roche), followed by incubation with polyclonal antibody raised against AlpMV in a rabbit, which was a gift from Dr. T. H. Chen [5]. To visualize antibody-specific proteins, the immunoblot was incubated with alkaline phosphatase (AP)-conjugated goat anti-rabbit IgG (ICN Pharmaceuticals, Ohio) at a 1:1000 dilution, and then exposed to a solution containing NBT (nitroblue tetrazolium chloride) and BCIP (5-bromo- 4-chloro-3-indolyl phosphate, toluidine salt) (Roche) in the AP substrate buffer (0.1 M Tris-HCl, pH 9.5, 0.05 M $\left.\mathrm{MgCl}_{2}, 0.1 \mathrm{M} \mathrm{NaCl}\right)$.

Sequence alignments were performed using Clustal W [20]. Phylogenetic analyses were carried out using programs in PHYLIP version 3.57c [10]. Genetic distances between pairs of amino acid sequences were calculated using PROTDIST (Dayhoff PAM). Phylogenetic trees were constructed by a distance method (FITCH) using the original data set and 1000 bootstrap data sets generated by the program SEQBOOT from the original set. The program TREEVIEW [16] was used to draw the phylogenetic tree.

A total of 1,731 nucleotides were determined from the representative cDNA clone alpcp-1 (GenBank accession number AF499025). Preliminary analysis of the sequence revealed the presence of an open reading frame ranging from nucleotide 1 to 1545, followed by an untranslated region of 186 nucleotides. A blast search of the databases found a match with the NIb and coat protein sequences characteristic of the family Potyviridae. To confirm that the alpcp-1 cDNA clone was derived from AlpMV, sequence encompassing the CP was amplified by PCR and cloned in pQE31 expression vector. Analysis of the recombinant proteins obtained from pExcp12 transformants by SDS-PAGE demonstrated that a protein band with molecular mass of the expected size appeared $1.5 \mathrm{~h}$ after induction with IPTG (Fig. 1A, lane 2), and still persisted $3 \mathrm{~h}$ after induction (Fig. 1A, lane 3 ). The identity of the expressed protein was confirmed by immunoblot analysis using the polyclonal antibody against AlpMV (Fig. 1B, lane 2).

A database search using the deduced amino acid sequence of AlpMV exhibited the highest identity to the corresponding region of CdMV (AF189125), followed by ChYNMV (AB044386), NLV (U58770), and MacMV (U58771); all of them belong to the genus Macluravirus. In order to find out sequences which are highly 


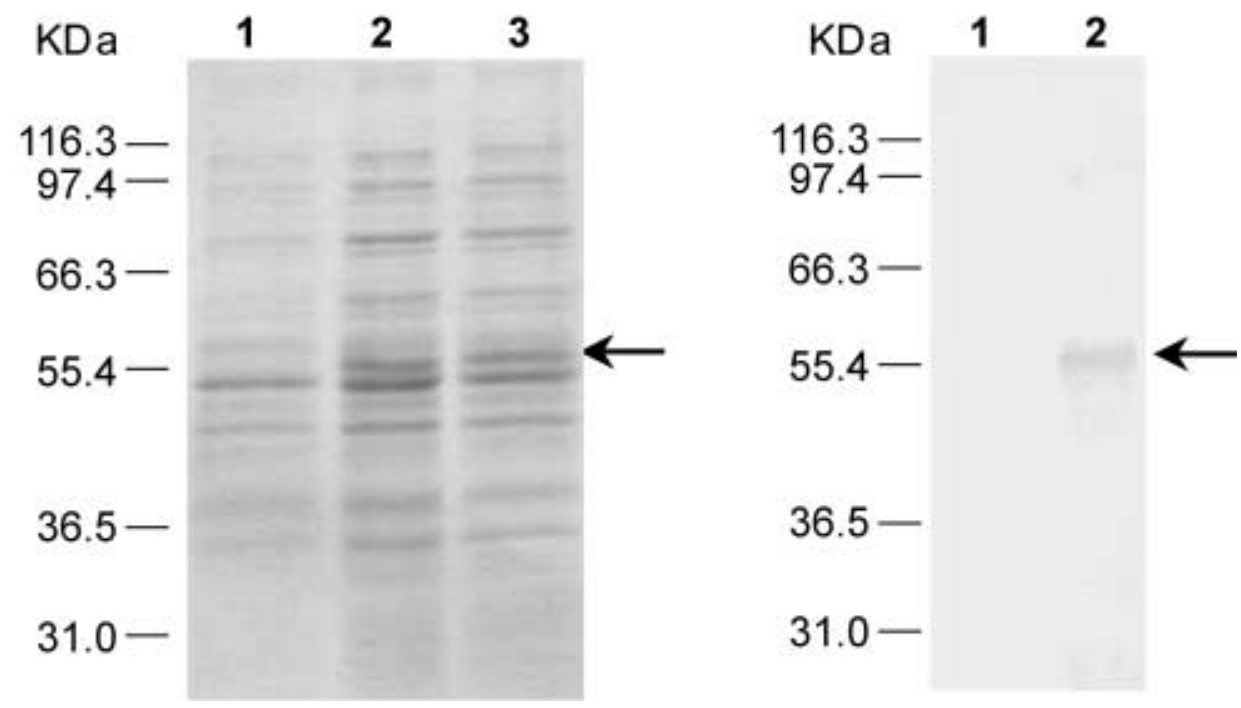

Fig. 1. SDS-polyacrylamide gel electrophoresis and immunoblot analysis of AlpMV coat protein expressed in E. coli. A Expression of the recombinant protein by M15[pREP4] cells harboring pExcp12 were induced with IPTG for indicated period of time and cell extract was analyzed by SDS-PAGE, followed by staining with coomassie blue. 1 : no induction, $2: 1.5 \mathrm{~h}$ after induction, $3: 3 \mathrm{~h}$ after induction. B Immunoblot analysis using polyclonal antibodies against AlpMV. 1: no induction, 2: $1.5 \mathrm{~h}$ after induction with IPTG. The position of the recombinant AlpMV CP is marked with an arrow. Positions of the molecular weight size marker are indicated on the left

Table 1. Coat protein amino acid sequence identity between AlpMV and selected members from the Potyviridae

\begin{tabular}{llcl}
\hline Genus & Virus & Identity $(\%)^{\mathrm{a}}$ & Accession number \\
\hline Macluravirus & MacMV & 42.9 & U58771 \\
& NLV & 46.9 & U58770 \\
& ChYNMV & 56.9 & AB044386 \\
& CdMV & 61.9 & AF189125 \\
Bymovirus & AlpMV & 100.0 & AF499025 \\
& BaYMV & 26.7 & X69757 \\
Ipomovirus & WSSMV & 25.8 & X73883 \\
& CVYV & 24.8 & AF233429 \\
Potyvirus & SPMMV & 24.7 & Z48058 \\
\multirow{3}{*}{ Rymovirus } & PVY & 30.3 & X68222 \\
Tritimovirus & TEV & 30.2 & M15239 \\
& AgMV & 30.9 & U30615 \\
& RGMV & 29.4 & U27383 \\
\hline
\end{tabular}

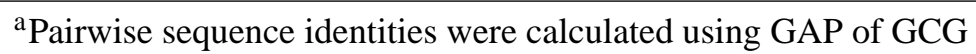


conserved among these viruses, the amino acid sequences of partial NIb and fulllength CP from NLV, MacMV, ChYNMV, CdMV, and AlpMV (Table 1) were analyzed by Clustal W. Alignments of the sequences demonstrated that NIb is highly conserved in several regions among these viruses (Fig. 2). The consensus motifs $(\mathrm{T} / \mathrm{S}) \mathrm{GX}_{3}-\mathrm{TX}_{3}-\mathrm{N}(\mathrm{T} / \mathrm{S})$ and GDD, proposed to be the active site for the RNA-dependent RNA polymerase of positive-strand RNA viruses [8, 15], were found at positions 4-14 and 47-49 of AlpMV, respectively. The possible NIa cleavage site between NIb and CP of NLV and MacMV has been suggested to be LQ/M by Badge et al [3]. As shown in Fig. 2, 'LQ/M' also exists in ChYNMV, but not in AlpMV and CdMV, which contained instead FQM at the corresponding position. If NIa cleavage indeed occurs after the glutamine residue (FQ/M), the $\mathrm{N}$-terminal residue of AlpMV CP will be methionine, the same as those of NLV, MacMV, and ChYNMV. Besides, it will generate a coat protein with a predicted molecular mass of $33.4 \mathrm{KDa}$, close to the size estimated for NLV (32.8 KDa) and MacMV (34.1 KDa) [3]. Attempts to determine the N-terminal sequence of the AlpMV CP by the Edman degradation method failed (data not shown), however, perhaps because methionine is relatively resistant to degradation compared to
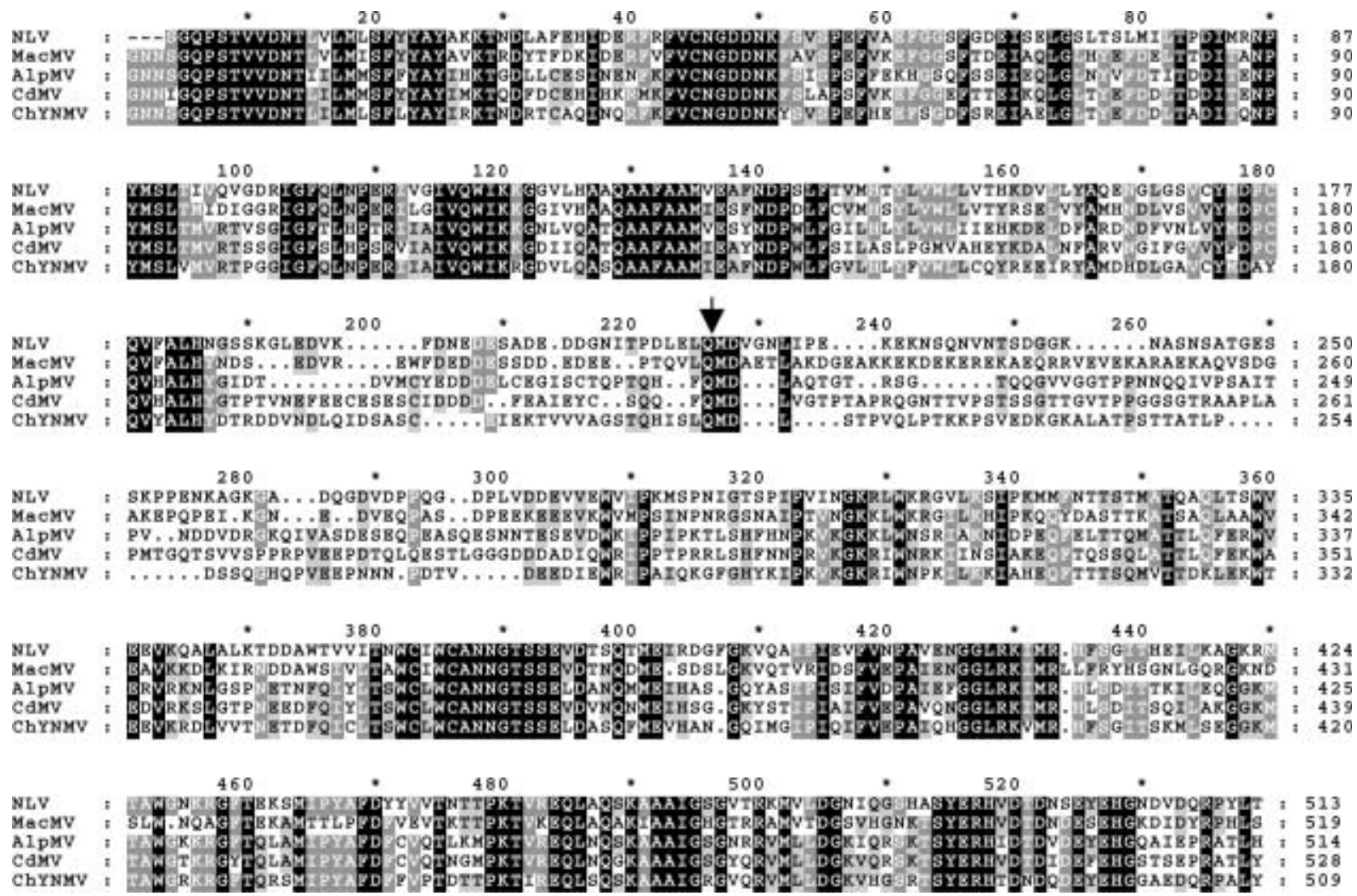

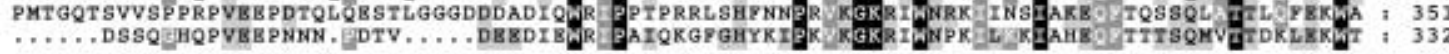

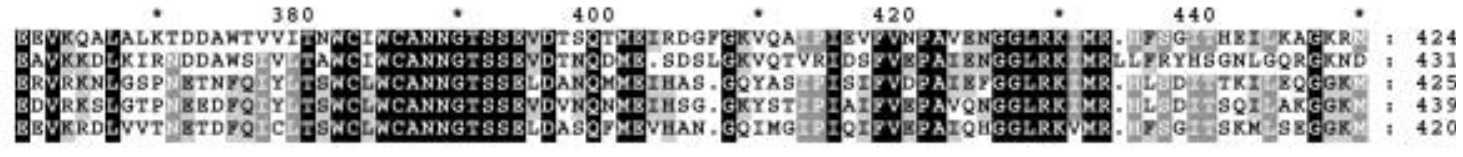

$\begin{array}{llllll}460 & * & 480 & * & 500 & 5\end{array}$

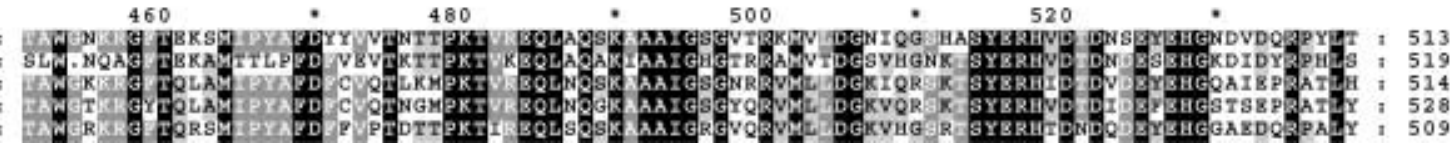

Fig. 2. Alignment of the partial ORFs of NLV (U58770), MacMV (U58771), CdMV (AF189125), ChYNMV (AB044386), and AlpMV (AF499025) by CLUSTAL W. The predicted NIa cleavage site is indicated with an arrow 


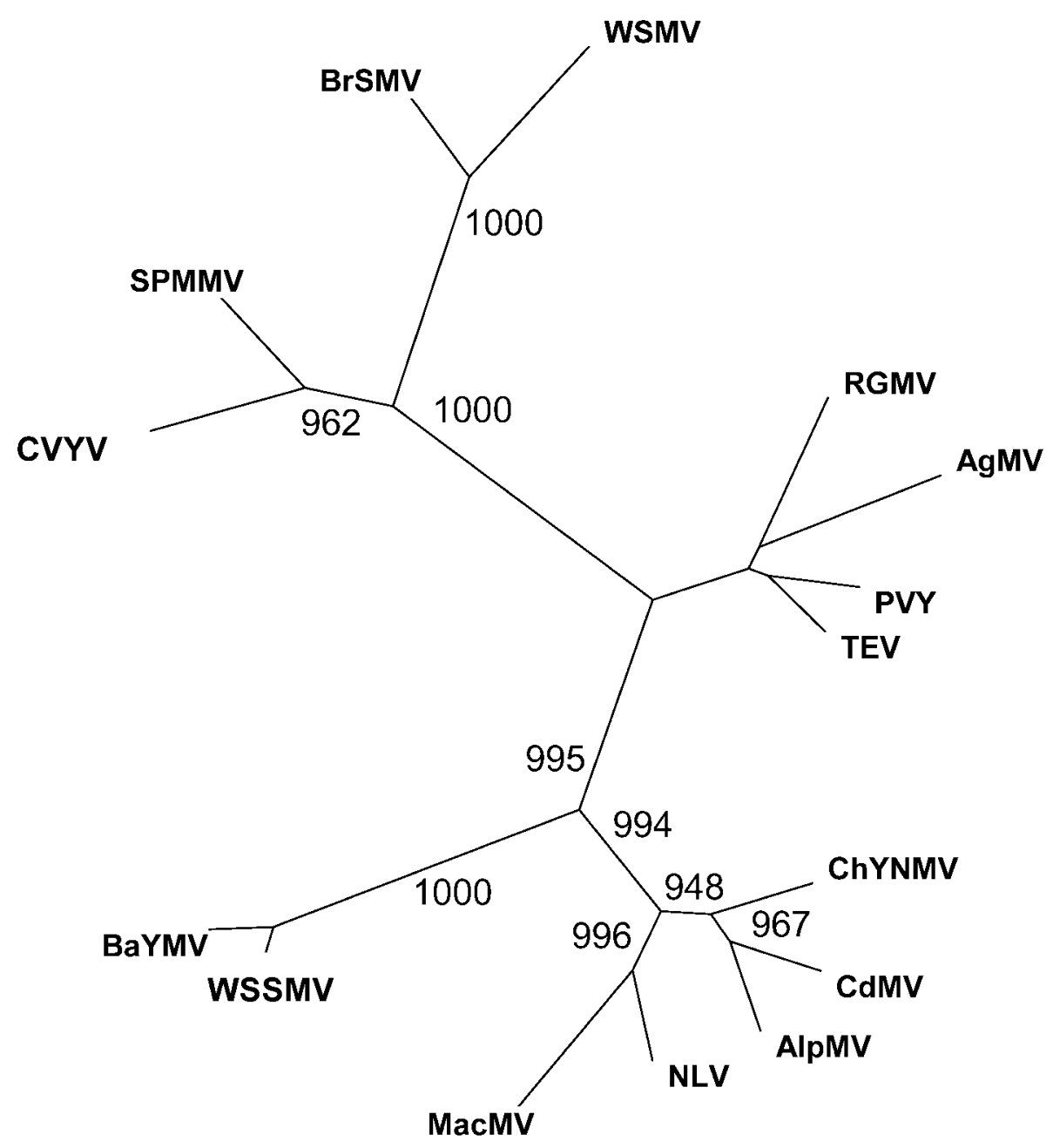

0.1

Fig. 3. Phylogenetic tree family for the amino acid sequences of the coat protein of AlpMV and selected members of the Potyviridae (Table 1). The tree was constructed using FITCH in PHYLIP. The values at the fork indicate the number of times out of 1000 trees that this grouping occurred after bootstrapping the data

other amino acid residues [6]. The deduced amino acid sequence of AlpMV CP had an estimated molecular mass of $33.4 \mathrm{KDa}$, whereas gel analysis of $\mathrm{CP}$ from purified virus suggested a molecular mass of $41 \mathrm{KDa}$ [5]. A similar discrepancy has been observed in NLV (32.8 KDa vs. 39.5 KDa) and MacMV (34.1 KDa vs. $40 \mathrm{KDa}$ ), and may be an artifact of the SDS-PAGE procedure [3]. 
As shown in Fig. 2, the C-terminus of viral CP is comparatively more conserved than the $\mathrm{N}$-terminal portion. A tripeptide motif, 'DAG', is highly conserved within a heptapeptide block at or near the $\mathrm{N}$ terminus of the coat protein of aphidtransmitted potyviruses $[1,2]$. Neither 'DAG' nor motifs similar to 'DAG' were found near the $\mathrm{N}$ terminus of the viral $\mathrm{CP}$ analyzed, although a 'DKG' tripeptide was found further downstream in the ChYNMV CP [14], and a 'DRG' was found in the AlpMV CP. The sequence 'NGTS', highly conserved in almost all potyviruses [17], was found in the context of 'WCANNGTSSE' in all five viruses (position 362-371 of AlpMV) (Fig. 2). Two highly conserved amino acid residues known to participate in virion assembly in potyvirids [13], $\mathrm{R}$ and $\mathrm{D}$, were found in the sequence of AlpMV ( $\mathrm{R}^{405}$ and $\mathrm{D}^{445}$ ) as well as other viral sequences analyzed. The sequence downstream of $\mathrm{D}^{445}$ of AlpMV is also highly conserved among the macluraviruses. The significance of this conservation awaits further investigation.

To investigate the relationship of AlpMV with other members of the Potyviridae, the deduced amino acid sequence of its coat protein was compared with those of 14 selected members of the family by GAP(GCG). The CP of AlpMV was $42.9 \sim 61.9 \%$ identical to that of members of the genus Macluravirus, but only $22.5 \sim 30.9 \%$ identical to members of other genera (Table 1). According to Shukla et al. [17], distinct species within the genus Potyvirus have coat protein sequence identity in the range of $38 \sim 71 \%$. Therefore, it is not appropriate to classify AlpMV as a species of the genus Potyvirus. Phylogenetic analyses of the coat protein amino acid sequences showed six clusters corresponding to the recognized genera and placed AlpMV in the same cluster as CdMV and ChYNMV. This demonstrates clearly that AlpMV could be recognized as a tentative member within the genus Macluravirus. As shown above, the 'DAG' motif essential for aphid transmission in the genus Potyvirus is not found in the CPs of macluraviruses, although similar motifs may be found downstream. Mutational analysis of CP or sequencing of the viral genome may provide information in regard to the mechanism of aphid transmission in the genus Macluravirus.

\section{GenBank accession number}

The nucleotide sequence obtained was deposited in GenBank with the accession number AF499025.

\section{Acknowledgement}

We thank Dr. T. H. Chen for supplying the polyclonal antibody against AlpMV, and Dr. Y. C. Chang for the potyvirus universal primer.

\section{References}

1. Atreya CD, Raccah B, Pirone TP (1990) A point mutation in the coat protein abolishes aphid transmission of a potyvirus. Virology 178: 161-165

2. Atreya PL, Lopez-Moya JJ, Chu MH, Atreya CD, Pirone TP (1995) Mutational analysis of the coat protein $\mathrm{N}$-terminal amino acids involved in potyvirus transmission by aphids. J Gen Viol 76: 265-270 
3. Badge J, Robinson DJ, Brunt AA, Foster GD (1997) 3'-terminal sequences of the RNA genomes of narcissus latent and maclura mosaic viruses suggest that they represent a new genus of the Potyviridae. J Gen Virol 78: 253-257

4. Brunt A, Crabtree K, Gibbs A (1990) Viruses of tropical plants. CAB International, UK

5. Chen TH, Hong JL (1996) Zingiberaceous ornamental mosaic- a new disease caused by an aphid-borne potyvirus in Taiwan. Plant Pathol Bull 5: 169-175

6. Dinant S, Lot H, Albouy K, Kuziak C, Meyer M, Astier-Manifacier S (1992) Nucleotide sequence of the $3^{\prime}$ terminal region of lettuce mosaic potyvirus RNA shows a Gln/Val dipeptide at the cleavage site between the polymerase and the coat protein. Arch Virol 116: $235-252$

7. Donald RG, Jackson AO (1994) The barley stripe mosaic virus $\gamma \mathrm{b}$ gene encodes a multifunctional protein that affects pathogenesis. Plant Cell 6: 1593-1606

8. Dougherty WG, Carrington JC (1988) Expression and function of potyviral gene products. Annu Rev Phytopathol 26: 123-143

9. Fauquet MC, Mayo MA (1999) Abbreviations for plant virus names-1999. Arch Virol 144: $1249-1272$

10. Felsenstein J (1993) PHYLIP (Phylogeny Inference Package) version 3.57c. Distributed by the author. Department of Genetics, University of Washington, Seattle

11. Gonsalves D, Trujkllo E, Hoch HC (1986) Purification and some properties of a virus associated with cardamom mosaic, a new member of the potyvirus group. Plant Dis 70: 65-69

12. Jacob T, Usha R (2001) 3'-terminal sequence analysis of the RNA genome of the Indian isolate of cardamom mosaic virus: a new member of the genus Macluravirus of Potyviridae. Virus Genes 23: 81-88

13. Jacquet C, Delecolle B, Raccah B, Lecoq H, Dunez J, Ravelonandro M (1998) Use of modified plum pox virus coat protein genes developed to limit heteroencapsidationassociated risks in transgenic plants. J Gen Viol 79: 1509-1517

14. Kondo T (2001) The 3 '-terminal sequence of Chinese yam necrotic mosaic virus genomic RNA: a close relationship with macluravirus. Arch Virol 146: 1527-1535

15. Kramer G, Argos P (1984) Primary structural comparison of RNA-dependent RNA polymerase from plant, animal and bacterial viruses. Nucleic Acids Res 12: 7269-7282

16. Page RDM (1996) TREEVIEW: An application to display phylogenetic tees on personal computers. Comput Appl Biosci 12: 357-358

17. Shukla DD, Ward CW, Brunt AA (1994) The Potyviridae. CAB International, Wallingford, UK

18. So IY (1980) Studies on ginger mosaic virus. Korean J Plant Prot 19: 67-72

19. Thomas JE (1986) Purification and properties of ginger chlorotic fleck virus. Annu Appl Biol 108: 43-50

20. Thompson JD, Higgins DG, Gibson TJ (1994) CLUSTAL W: improving the sensitivity of progressive multiple sequence alignment through sequence weighting, position-specific gap penalties and weight matrix choice. Nucleic Acids Res 22: 4673-4680

Author's address: Dr. Ruey-Fen Liou, Department of Plant Pathology, National Taiwan University, \#1, Sec. 4, Roosevelt Road, Taipei 106, Taiwan; e-mail: rfliou @ccms.ntu.edu.tw 